\title{
RADIATIVE CORRECTIONS TO WW SCATTERING
}

\author{
M.J.G. VELTMAN
}

Randall Laboratory of Physics, University of Michigan, Ann Arbor, MI 48109, USA

\section{F.J. YNDURẢIN}

Departamento de Física Teórica, Universidad Autónoma de Madrid, E-28049 Madrid, Spain

Received 15 March 1989

\begin{abstract}
The one-loop radiative corrections to vector-boson-vector-boson scattering are calculated in the limit of high energy and even higher Higgs mass. To parametrize the limit of large Higgs mass a new particle, the U-particle is introduced.
\end{abstract}

\section{Introduction}

Few people would question the assertion that the least understood part of the Standard Model is the Higgs mechanism. The Higgs particle is necessary for the renormalizability of the theory but this in itself is a somewhat empty statement: a Higgs with a mass of, say, $10^{19} \mathrm{GeV}$ is supposedly very nearly the same thing as no Higgs at all. One may ask what would the physical consequences be of a very heavy Higgs particle, perhaps not as heavy as the Planck mass but much heavier than $M$, where $M$ is the $\mathrm{W}$ or $\mathrm{Z}$ mass. It has been known since ancient times that such a heavy Higgs implies a breakdown of perturbation theory; but as far as low energy observable consequences are concerned (low energy here means $s \leqq M^{2}$ ) a screening theorem [1] seems to be in operation that makes the Higgs' absence go (almost) unnoticed ${ }^{\star}$. As far as we know, upper bounds on $m_{\mathrm{H}}$ are not particularly meaningful, being based upon perturbation theory which, precisely, fails if $m_{\mathbf{H}}$ is large.

Screening disappears at higher energies, in particular in WW scattering which may become observable with future or even present accelerators. When $s$ (the CM energy squared) gets larger than $M^{2}$, the amplitude for scattering ${ }^{\star \star}$ of longitudinally polarized W's grows like $s$, at tree level, and this growth continues until the mass of the Higgs is reached; thereafter, the amplitude stays constant at a value

\footnotetext{
* In the one-loop approximation.

$\star \star$ To avoid unessential complications, we work in the approximation in which the weak angle vanishes and thus we have three W's of equal mass.
} 
$\propto m_{\mathrm{H}}^{2}$. This has led some people to suggest an upper bound for $m_{\mathrm{H}}^{2}$ because, if it were too large, the amplitude would violate the unitarity bound $[2,3]$. To be precise, if we denote isospin indices by $a, b, c, d$ varying from 1 to 3 , we have, for $s, t, u \gg M^{2}$ but $m_{\mathrm{H}}^{2} \gg s, t, u$,

$$
F(a+b \rightarrow c+d) \simeq \frac{g^{2}}{16 \pi^{2} M^{2}}\left(s \delta_{a b} \delta_{c d}+t \delta_{a d} \delta_{b c}+u \delta_{a c} \delta_{b d}+\text { constant }\right),
$$

where $s, t, u$ are the standard Mandelstam variables, and $F$ is normalized so that, for $a=b=c=d$,

$$
F=2 ! \frac{2 \sqrt{s}}{\pi|k|} \sum_{l=\text { even }}(2 l+1) P_{l}(\cos \theta) \sin \delta_{l} \mathrm{e}^{i \delta_{l}} .
$$

The 2 ! comes from the identity of particles, and $\boldsymbol{k}$ is the CM momentum, $|\boldsymbol{k}| \simeq \frac{1}{2} \sqrt{s}$ when $s \gg M^{2}$. Eq. (1) is valid at the tree level; the unitarity limit is reached at $s^{1 / 2} \simeq 4 M / \alpha_{\mathrm{W}}^{1 / 2} \simeq 2 \mathrm{TeV}$. Of course, this violation of unitarity does not take place or, if unitarity is violated, it will happen in a less brutal way. This is seen clearly if we iterate each of the terms in eq. (1). For example, at the one-loop level, the piece

$$
\frac{g^{2}}{16 \pi^{2} M^{2}} s \delta_{a b} \delta_{c d}
$$

of eq. (1) is multiplied by a term

$$
1+\frac{3 \alpha_{\mathrm{W}} s}{32 \pi M^{2}} \log \frac{m_{\mathrm{H}}^{2}}{-s}+\cdots,
$$

where the dots represent terms of lower order in $s$. Iterating, the sum of the whole string of sausages becomes

$$
F \sim \delta_{a b} \delta_{c d} \frac{g^{2}}{16 \pi^{2} M^{2}} \frac{s}{1-\left(3 \alpha_{\mathrm{W}} s / 32 \pi M^{2}\right) \log m_{\mathrm{H}}^{2} /(-s)}+\cdots,
$$

where the dots represent terms suppressed, order by order, by powers of $\log m_{\mathrm{H}}^{2} /(-s)$. Now expression (3) stays perfectly inside the unitarity limit; the only effect a large Higgs mass has is a broad enhancement: the partial wave amplitude approaches the unitarity limit around

$$
\frac{3 \alpha_{\mathrm{W}} s}{32 \pi M^{2}} \log \left|\frac{m_{\mathrm{H}}^{2}}{s}\right| \simeq 1,
$$

i.e., at $s^{1 / 2} \sim 2.4 \mathrm{TeV} /\left|\log m_{\mathrm{H}}^{2} / s\right|^{1 / 2}$, and then decreases logarithmically. 
Life would be easy if there were no more than this. Unfortunately, besides the terms $s^{2} \log m_{\mathrm{H}}^{2} / s$ like the one in expression (2) there are, already at the one-loop level, corrections of the type $t^{2} \log m_{\mathrm{H}}^{2} / t$, and mixed ones, so the summation procedure of expression (3) is not enough. If we had an $\mathrm{SU}(N)$ theory with $N$ large we could get away with this, for only the term in $\log m_{\mathrm{H}}^{2} / s$ has a coefficient growing with $N$ (indeed, the 3 in expression (2) is in reality $N^{2}-1$ ); in the large $N$ limit, expression (3) would be exact, a fact already known [4]. Since in the real world $N=2$, a not particularly large number, the full one-loop corrections have to be calculated. Nevertheless, the argument leading to expression (3) indicates that a perturbation-theoretic violation of unitarity does not necessarily indicate a sick theory. In this spirit, we present here a calculation of the one-loop corrections to longitudinal WW scattering [5,6], in the kinematic region $M^{2} \ll s, t, u \ll m_{\mathrm{H}}^{2}$. At worst, they will serve as a refinement of the tree-level calculation; at best, they may lead to further understanding of the theory in the limit of large Higgs mass, and even, with luck, help discover new physics.

Besides the ordinary $\mathrm{W}$ - and Higgs particles, and their interactions, we will introduce a scalar particle that we will call $\mathrm{U}$, assumed to be a singlet under $\mathrm{SU}(2)$ and therefore not coupled to the $\mathrm{W}$. The $\mathrm{U}$ allows us to control the limit of very large Higgs mass in the following way. If we couple $U$ to the Higgs with a strength proportional to $m_{\mathrm{H}}^{2}$, then it will still contribute to WW scattering at the one-loop level with terms quadratic in $s, t, u$.

If $m_{\mathrm{U}} \sim m_{\mathrm{H}}$, then the $\mathrm{U}$ contributes to nonlogarithmic terms; but if its mass is small, say $m_{\mathrm{U}} \sim M$, it will yield terms $\dot{s}^{2} \log s$ as well. If $m_{\mathrm{U}}=m_{\mathrm{H}}$ and we let $m_{\mathrm{H}} \rightarrow \infty$, the $\mathrm{U}$ particle becomes utterly invisible: but with its inclusion the one-loop corrections to WW scattering can be altered. This shows clearly arbitrariness in the limit $m_{\mathrm{H}} \rightarrow \infty$.

Besides its use as a theoretical artifact, the $\mathrm{U}$ stands for objects that only couple to ordinary matter via the Higgs (and presumably gravity). If $m_{U}^{2} \ll s$ the $U$ may be produced really (and copiously) in WW collisions. The one-loop corrections to this process could well be the only indications of the existence of U-like particles, other than their gravitational effects. There is a large domain of speculation here that we prefer to leave to the appropriate experts ${ }^{\star}$.

\section{The model}

The model chosen is a simplified version of the Standard Model. Electromagnetism is not considered (the weak mixing angle is taken to be zero), and also

\footnotetext{
${ }^{\star}$ See the proceedings of any conference on dark matter or other exotic subjects.
} 
fermions are ignored. The gauge group is thus $\mathrm{SU}_{2}$, and the invariant lagrangian is

$$
\begin{aligned}
\mathscr{L}_{\mathrm{inv}}= & -\frac{1}{4} G_{\mu \nu}^{a} G_{\mu \nu}^{a}-\frac{1}{2} M^{2} W^{2}-\frac{1}{2}\left(\partial_{\mu} H\right)^{2}-\frac{1}{2} m^{2} H^{2}-\frac{1}{2}\left(\partial_{\mu} \varphi^{a}\right)\left(D_{\mu}^{a} \varphi\right) \\
& +\frac{1}{2} g W_{\mu}^{a}\left(H \partial_{\mu} \varphi^{a}-\varphi^{a} \partial_{\mu} H\right)-\frac{1}{8} g^{2} W_{\mu}^{2}\left(\varphi^{2}+H^{2}\right)-\frac{1}{2} g M W^{2} H \\
& -r M g H\left(\varphi^{2}+H^{2}\right)-\frac{1}{8} r g^{2}\left(\varphi^{2}+H^{2}\right)^{2}-M \varphi^{a} \partial_{\mu} W_{\mu}^{a}-\frac{1}{2}\left(\partial_{\mu} U\right)^{2} \\
& -\frac{1}{2} m_{\mathrm{u}}^{2} U^{2}-g g_{\mathrm{u}} r M U^{2} H-\frac{1}{4} g^{2} g_{\mathrm{u}} r U^{2}\left(H^{2}+\varphi^{2}\right),
\end{aligned}
$$

$r=m^{2} / 4 M^{2}, H$ is the Higgs field, and $\varphi^{a}$ the Higgs ghost triplet.

The reader may note that we have added a scalar singlet field $U$, coupled in a gauge invariant way to the Higgs system, i.e., as $\left(H^{2}+\varphi^{2}\right) U^{2}$ before symmetry breakdown. Two choices for the mass of the U-particle have been considered, namely $m_{\mathrm{u}}=m$ (Higgs mass) and $m_{\mathrm{u}}=M$ (vector boson mass). The coupling has been chosen such that it resembles closely the Higgs self-coupling. The $U$ field, and the associated parameter $g_{u}$ may be understood as a mechanism to specify or parametrize the behaviour of the theory in the limit of a heavy Higgs. Any dependence on $g_{\mathrm{u}}$ implies sensitivity to details of the way the limit of heavy Higgs is taken.

It should be noted that the particular choices made here are by no means the only ones. The range of possibilities and the physics aspects of this Higgs-U (for unknown) system are not investigated in this article.

The above lagrangian must be supplemented with a gauge breaking term and a ghost lagrangian. We will work in the Feynman-'t Hooft gauge, and the resulting Feynman rules are given in appendix A.

\section{The tree amplitude}

The amplitude for longitudinally polarized vector boson scattering has been studied before. In particular, Lee et al. [3] have considered this amplitude in the limit of infinite $s$ (= center-of-mass energy squared). We will generally work in the approximation of large $s$ (and $t$ and $u$ ), but even larger Higgs mass:

$$
M^{2} \ll s \ll m^{2},
$$

where $M$ and $m$ are vector boson and Higgs mass respectively.

Calculating the tree amplitude is a simple matter. The notation used is defined in fig. 1.

All momenta are taken to be ingoing. The indices $a, b, c$ and $d$ denote the isospin state of the W's. The amplitude must be multiplied by four polarization vectors $e_{\alpha}(k), e_{\beta}(p)$, and $e_{\gamma}\left(p^{\prime}\right)$ and $e_{\delta}\left(k^{\prime}\right)$ with $k_{\alpha} e_{\alpha}(k)=0$, etc. We specialize to 


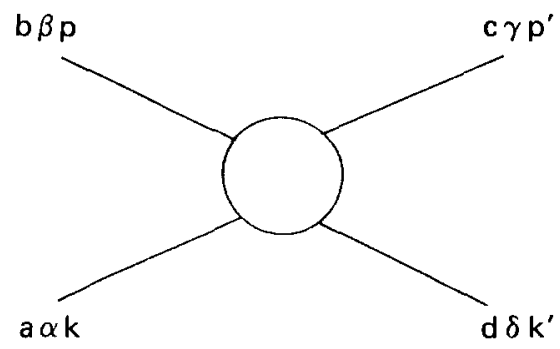

Fig. 1. Notation.

longitudinally polarized W's. In the center-of-mass system, with $\boldsymbol{p}$ and $\boldsymbol{k}$ along the third axis and $\boldsymbol{p}^{\prime}$ and $\boldsymbol{k}^{\prime}$ in the 1-3 plane one has

$$
\begin{aligned}
k & =\left(0,0, k_{l}, i k_{0}\right), \quad k^{\prime}=-\left(k_{l} \sin \theta, 0, k_{l} \cos \theta, i k_{0}\right), \\
e_{\alpha}(k) & =\frac{1}{M}\left(0,0, k_{0}, i k_{l}\right), \\
e_{\delta}\left(k^{\prime}\right) & =-\frac{1}{M}\left(k_{0} \sin \theta, 0, k_{0} \cos \theta, i k_{l}\right),
\end{aligned}
$$

and similarly for $p$ and $p^{\prime}$ differing from the above by a minus sign for the spatial components.

Ignoring terms that behave as a constant for large $s, u$ or $t$ the result is

$$
\begin{aligned}
A_{\mathrm{wW}}=i g^{2}(2 \pi)^{4}[ & \delta_{a b} \delta_{c d}\left(\frac{s}{4 M^{2}}+\frac{s^{2}}{4 M^{2}} \frac{1}{-s+m^{2}}\right) \\
& +\delta_{a c} \delta_{b d}\left(\frac{u}{4 M^{2}}+\frac{u^{2}}{4 M^{2}} \frac{1}{-u+m^{2}}\right) \\
& \left.+\delta_{a d} \delta_{b c}\left(\frac{t}{4 M^{2}}+\frac{t^{2}}{4 M^{2}} \frac{1}{-t+m^{2}}\right)\right]
\end{aligned}
$$

In here, as usual,

$$
\begin{gathered}
s=-(p+k)^{2}, \quad t=-\left(p+p^{\prime}\right)^{2}, \quad u=-\left(k+p^{\prime}\right)^{2}, \\
s+t+u=4 M^{2} .
\end{gathered}
$$

If $m^{2} \gg|s|,|t|,|u|$ then the Higgs exchange terms may be ignored and the amplitude grows with $s$ (or $u$ or $t$ ). If the Higgs mass $m<s$ then the Higgs exchange terms tend to cancel the other terms and the amplitude levels off to a 
constant. This constant is $i g^{2}(2 \pi)^{4} m^{2} / 4 M^{2}$. The previously mentioned unitarity limit $[2,3]$ is based on this constant.

It should be noted that the amplitude involves already one cancellation due to the gauge structure of the theory. For example, if there were no four-W vertex the amplitude would behave as $s^{2}$, no matter what Higgs mass.

The fact that there is such a cancellation makes the validity of the equivalence theorem [7] in this case not transparent. This theorem states that the longitudinal W scattering amplitude equals the $\varphi-\varphi$ ( $\varphi$ is the Higgs ghost) scattering amplitude up to terms $M^{2} / s$. Because of the cancellation mentioned the amplitude is of the same order as the terms neglected. However, explicit calculation shows that the $\varphi-\varphi$ amplitude equals the longitudinal $\mathrm{W}-\mathrm{W}$ amplitude, in accordance with the equivalence theorem

\section{One-loop renormalization}

The renormalization scheme used is the standard one, as explained in detail in ref. [8]. In that reference terms proportional to the Higgs mass squared were included in the renormalization scheme. Here we will go slightly further and include terms proportional to the logarithm of the Higgs mass. For the WW and WWW amplitude such terms were computed a decade ago [1]; here we need a few more amplitudes.

As a first step one must calculate

(i) all infinities (poles in $n-4$ ) of all two- and three-point functions;

(ii) all terms behaving like $m^{2}$ or $\log m^{2}$ for all two- and three-point functions;

(iii) the complete set of tadpole diagrams.

The result of this work can conveniently be described as follows. Make in the invariant lagrangian only (thus not in the gauge fixing part) the replacements

$$
\begin{array}{rlrl}
W_{\mu}^{a} \rightarrow W_{\mu}^{a}\left(1+\delta_{W}\right), & g \rightarrow g\left(1+\delta_{g}\right), \\
\varphi \rightarrow \varphi\left(1+\delta_{H}\right), & H \rightarrow H\left(1+\delta_{H}\right)+\frac{M}{g} \delta_{t}, \\
M & \rightarrow M\left(1+\delta_{M}\right), & m \rightarrow m\left(1+\delta_{m}\right) .
\end{array}
$$

Now the various $\delta$ can be chosen such that they reproduce in a tree-level calculation exactly the terms (i)-(iii). Giving such terms a minus sign would amount to cancellation of those terms.

Instead of $\delta_{M}$ and $\delta_{m}$ we use $\delta_{1}$ and $\delta_{2}$ defined by

$$
\delta_{1}=\delta_{M}+\frac{1}{2} \delta_{t}, \quad \delta_{2}=\delta_{M}-\delta_{m} .
$$


The terms (i)-(iii) are reproduced if we take

$$
\begin{aligned}
& \delta_{t}= \frac{i \pi^{2} g^{2}}{n-4}\left(\frac{3}{2}+9 \frac{M^{2}}{m^{2}}+\frac{3}{2} \frac{m^{2}}{M^{2}}+\frac{1}{2} \frac{m^{2}}{M^{2}} g_{\mathrm{u}}+\frac{1}{2} g_{\mathrm{u}}^{\prime}\right) \\
&+i \pi^{2} g^{2}\left(-\frac{3}{4}-\frac{3}{2} \frac{M^{2}}{m^{2}}+\frac{9}{2} \frac{M^{2}}{m^{2}} \log M^{2}-\frac{3}{4} \frac{m^{2}}{M^{2}}+\frac{3}{4} \frac{m^{2}}{M^{2}} \log m^{2}\right. \\
&\left.+\frac{3}{4} \log M^{2}-\frac{1}{4} \frac{m^{2}}{M^{2}} g_{\mathrm{u}}+\frac{1}{4} \frac{m^{2}}{M^{2}} g_{\mathrm{u}} \log m^{2}-\frac{1}{4} g_{\mathrm{u}}^{\prime}+\frac{1}{4} g_{\mathrm{u}}^{\prime} \log M^{2}\right), \\
& \delta_{1}=-\frac{25 i \pi^{2}}{6} \frac{g^{2}}{n-4}+i \pi^{2} g^{2}\left(\frac{1}{16} \frac{m^{2}}{M^{2}}+\frac{5}{12} \log m^{2}\right), \\
& \delta_{2}= \frac{i \pi^{2} g^{2}}{n-4}\left(-\frac{8}{3}-\frac{9}{2} \frac{M^{2}}{m^{2}}-\frac{3}{2} \frac{m^{2}}{M^{2}}-\frac{1}{8} \frac{m^{2}}{M^{2}} g_{\mathrm{u}}^{2}-\frac{1}{8} \frac{m^{2}}{M^{2}} g_{\mathrm{u}}^{\prime 2}\right) \\
&+i \pi^{2} g^{2}\left(-\frac{3}{2}+\frac{7}{16} \frac{m^{2}}{M^{2}}-\frac{3}{4} \frac{m^{2}}{M^{2}} \log m^{2}+\frac{7}{6} \log m^{2}-\frac{9}{4} \frac{M^{2}}{m^{2}} \log m^{2}\right. \\
&\left.-\frac{9}{16} \frac{m^{2}}{M^{2}} B_{0}-\frac{1}{16} \frac{m^{2}}{M^{2}} g_{\mathrm{u}}^{2} \log m^{2}-\frac{1}{16} \frac{m^{2}}{M^{2}} g_{\mathrm{u}}^{2} B_{0} \log m^{2}+\frac{1}{8} \frac{m^{2}}{M^{2}} g_{\mathrm{u}}^{\prime 2}\right), \\
& \delta_{\mathrm{g}}=-\frac{43 i \pi^{2}}{6} \frac{1}{n-4} \cdot \\
& \delta_{\mathrm{W}}=i \pi^{2} g^{2}\left(\frac{19}{6} \frac{1}{n-4}-\frac{1}{24} \log m^{2}\right), \\
& \pi^{2}\left(\frac{3}{2} \frac{1}{n-4}+\frac{m^{2}}{16 M^{2}}+\frac{1}{8} \log m^{2}\right),
\end{aligned}
$$

In here $B_{0}$ is the two-point function

$$
\frac{1}{i \pi^{2}} \int \mathrm{d}_{4} q \frac{1}{\left(q^{2}+M^{2}\right)\left[(q+p)^{2}+M^{2}\right]}
$$


on mass shell, i.e., $p^{2}=-M^{2}$. One has

$$
B_{0}=\frac{\pi}{\sqrt{3}}-2
$$

The terms with $g_{\mathrm{u}}$ and $g_{\mathrm{u}}^{\prime}$ are the contributions if $m_{\mathrm{u}}=m$ and $m_{\mathrm{u}}=M$ respectively. The result for $\delta_{2}$ needs some discussion. It relates directly to the way the Higgs propagator is renormalized. We have chosen $\delta_{2}$ in such a way that the one-loop results together with counterterms (i.e., the $\delta$ with a minus sign) display for the Higgs propagator an unchanged mass value.

It should be noted that there is no $\log m^{2}$ term in the coupling renormalization factor $\delta_{g}[1]$.

With counterterms as defined by the above no $\log m^{2}$ dependence remains for the two- or three-point $\mathrm{W}$ functions. As shown in ref. [1], dependence on $\log m^{2}$ shows up elsewhere (after renormalization). It is interesting to note what happens with the four-W amplitude. Computing the $\log m^{2}$ terms, and subtracting the $\log m^{2}$ terms in $2 \delta_{\mathrm{g}}+4 \delta_{\mathrm{W}}$ one finds a non-zero result. Specializing to longitudinally polarized W's this result is

$$
\begin{aligned}
A_{\mathrm{wwww}} \simeq \frac{i \pi^{2}}{M^{4}} \log m^{2}[ & \delta_{a b} \delta_{c d}\left(\frac{1}{24} s t+\frac{1}{96} s^{2}+\frac{1}{24} t^{2}\right) \\
& \left.+\delta_{a c} \delta_{b d}\left(\frac{1}{24} s u+\frac{1}{96} u^{2}+\frac{1}{24} s^{2}\right)+\delta_{a d} \delta_{b c}\left(\frac{1}{24} t u+\frac{1}{96} t^{2}+\frac{1}{24} u^{2}\right)\right] .
\end{aligned}
$$

The calculation is done for one-particle irreducible diagrams only, keeping only terms proportional to $\log m^{2}$.

Since the renormalized self-energy and vertex part contain no $\log m^{2}$ dependence one might think that the above amplitude gives precisely all $\log m^{2}$ for the full amplitude (irreducible plus reducible diagrams). This, however, is not true. Diagrams of the type shown in fig. 2 give a non-vanishing contribution even in the limit of large Higgs mass. The $1 / \mathrm{m}^{2}$ behaviour of the propagators is offset by $\mathrm{m}^{2}$ dependence in the vertex and self-energy diagrams.
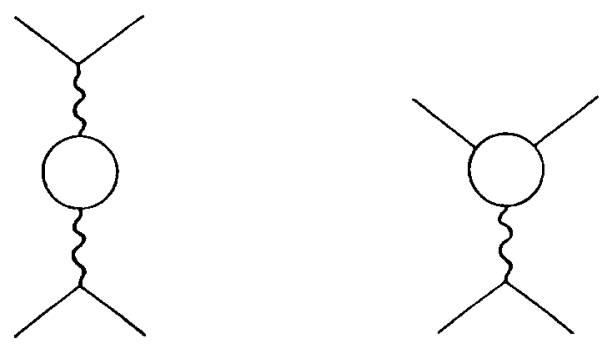

Fig. 2. Non-vanishing diagrams for large $m$. 
This is quite unsettling, especially for the second type of diagram. After all, renormalization was done such that the pole of the renormalized propagator is unchanged.

It is really very difficult to argue further on these contributions. Are they real, in the sense that they represent what happens in a theory without Higgs? One cannot arbitrarily neglect these terms, as that would upset gauge invariance. It is because of this situation that we have introduced the U-particle. That particle truly represents a variation, a new parameter in the way the limit of large Higgs mass is taken.

The terms resulting from the diagrams of fig. 2 modify the above quoted result for $A_{\text {wwww }}, \log m^{2}$ part such that the coefficients $\frac{1}{96}$ occurring in the above become $\frac{1}{24}$.

At this point it is of interest to discuss a remarkable fact, pointed out to us by Bouamrane [9]. The remark is that of all quantities $\delta$ computed and shown above only $\delta_{2}$ (essentially Higgs mass renormalization) is of any consequence to the four-W amplitude.

This fact is essentially due to the cancellation of terms of second order in $s, t$ and $u$ in the tree amplitude. More precisely this can be understood in the following manner. Write the scattering amplitude for longitudinal W's, to fourth order in $g$ and for $k^{2} \gg M^{2}$.

$$
F=\frac{C_{2}}{M^{2}} g^{2}+\frac{C_{4}}{M^{4}} g^{4}+\cdots
$$

We leave out the Higgs contributions for the moment. The coefficients $C_{2}, C_{4}$ depend on $s, t$ and $u$. When renormalizing we may replace $M^{2}$ by $M^{2}\left(\mu^{2}\right), g^{2}$ by $g^{2}\left(\mu^{2}\right)$, where $\mu^{2}$ parametrizes the renormalization. $C_{2}$, being a tree-diagram coefficient, is independent of $\mu^{2}$. As for $C_{4}$, we may split it:

$$
C_{4}=C_{4}^{(2)}\left(\mu^{2}\right)+C_{4}^{(1)}\left(\mu^{2}\right) \text {, }
$$

where $C_{4}^{(2)}$ is quadratic in $s, t$ and $u$, and $\mathrm{C}_{4}^{(1)}$ is linear. The structure of $F$ is then

$$
F=\frac{C_{2}}{M^{2}\left(\mu^{2}\right)} g^{2}\left(\mu^{2}\right)+\frac{C_{4}^{(2)}\left(\mu^{2}\right)}{M^{4}} g^{4}+\frac{C_{4}^{(1)}\left(\mu^{2}\right)}{M^{4}} g^{4}+\cdots,
$$

where we have taken $M^{4}, g^{4}$ independent of $\mu^{2}$ in the last two terms. This is due to the fact that an alteration of $\mu^{2}$ in $M^{2}\left(\mu^{2}\right)$ or $g^{2}\left(\mu^{2}\right)$ induces changes of higher order only.

If we alter $\mu^{2} \rightarrow \mu^{\prime 2}$ then, because the ratio $g^{2}\left(\mu^{2}\right) / M^{2}\left(\mu^{2}\right)$ is not renormalization invariant we will get

$$
\frac{C_{2}}{M^{2}\left(\mu^{2}\right)} g^{2}\left(\mu^{2}\right) \rightarrow \frac{C_{2}}{M^{2}\left(\mu^{\prime 2}\right)} g^{2}\left(\mu^{\prime 2}\right)+\frac{C_{2}}{M^{2}} \lambda g^{4},
$$


where $\lambda$ is defined by

$$
\frac{g^{2}\left(\mu^{\prime 2}\right)}{M^{2}\left(\mu^{\prime 2}\right)}=\frac{g^{2}\left(\mu^{2}\right)}{M^{2}\left(\mu^{2}\right)}\left(1+\lambda g^{2}\right)
$$

Since $F$ is observable it should not get altered by this. Therefore, the term $C_{2} \lambda g^{4} / M^{2}$ above should be cancelled by a similar term elsewhere in $F$. Now we must remember that $C_{2}$ is linear in $s, t$ and $u$ : $C_{2} \lambda g^{4} / M^{2}$ can only cancel against a variation of $C_{4}^{(1)}\left(\mu^{2}\right) g^{2} / M^{4}$, which is the term linear in $s, t$ and $u$. It then follows that $C_{4}^{(2)}$ has to be independent of $\mu^{2}$, as its variation cannot be cancelled by any other term. The renormalization for the leading (in terms of powers of $s, t$ and $u$ ) one-loop term shall cancel, as indeed verified by explicit calculations.

The argument is not valid for the Higgs contributions. At the tree level the contributions to $F$ of the Higgs propagator term are of the form

$$
F_{\mathrm{H}}=a \frac{s^{2}}{-s+m^{2}} g^{2}+\text { similar terms }
$$

When renormalizing, $m^{2} \rightarrow m^{2}+\delta m^{2}$, with $\delta m^{2}$ of order $g^{2}$. Thus

$$
\begin{aligned}
F_{\mathrm{H}} & =a \frac{s^{2}}{-s+m^{2}+\delta m^{2}} g^{2}+\cdots \\
& =a \frac{s^{2}}{-s+m^{2}} g-a \frac{s^{2} \delta m^{2}}{\left(-s+m^{2}\right)^{2}} g^{2}+\cdots
\end{aligned}
$$

It so happens that $\delta m^{2}$ contains terms quadratic in $\mathrm{m}^{2}$ :

$$
\delta m^{2}=c m^{4} \frac{g^{2}}{M^{2}}+\cdots,
$$

so that

$$
F_{\mathrm{H}}=a \frac{s^{2}}{-s+m^{2}} g^{2}-a c \frac{m^{4} s^{2}}{M^{2}\left(-s+m^{2}\right)^{2}} g^{4}+\cdots,
$$

or, in the limit $s \ll m^{2}$,

$$
F_{\mathrm{H}} \simeq a \frac{s^{2}}{m^{2}} g^{2}-a c \frac{s^{2}}{M^{2}} g^{4}+\cdots,
$$

showing that the Higgs mass renormalization contributes to terms $g^{4} s^{2}$. 


\section{Four-W amplitude}

The calculation of the one-loop amplitude for four longitudinally vector bosons is rather complicated and it is mandatory to have some checks on the answer. For this reason we have done three different calculations:

(i) An approximate dispersive calculation. Using the tree amplitude and specializing to the $s$-channel (i.e., taking $a=b$ and $c=d$ and $a \neq c$ in the equation for the three amplitude) the one-loop contribution modulo subtraction can be determined using a dispersion equation.

(ii) Calculation of the WWWW one-loop graphs, reducible plus irreducible.

(iii) Assuming the validity of the equivalence theorem in this one-loop calculation, and observing that there is no cancellation in the leading terms the four- $\varphi$ amplitude should give the same result as (ii). Thus, we calculated the $\varphi \varphi \varphi \varphi$ one-loop graphs, reducible plus irreducible.

All results (eventually) agreed. Fig. 3 shows the topologies included in the calculations (ii) and (iii). To the diagrams of fig. 3 must be added diagrams that are obtained from those of fig. 3 by crossing. The set of fig. 3 is the $t$-channel set. The $s$-channel set obtains by exchanging $p$ and $k^{\prime}$, and the $u$-channel set by exchanging $p^{\prime}$ and $k^{\prime}$.

From the result one must subtract the counterterms with structure as shown in fig. 4. To the diagrams in brackets the appropriately crossed diagrams must be added. The crosses represent factors $\delta_{g}$, etc. For example, one has for the first diagram, four-W amplitude $2 \delta_{\mathrm{g}}+4 \delta_{\mathrm{W}}$, in case of the four- $\varphi$ amplitude a factor $2 \delta_{\mathrm{g}}+4 \delta_{\mathrm{H}}-2 \delta_{2}$. The $\delta_{2}$ is related to the factor $\mathrm{m}^{2} / M^{2}$ in the vertex.

Throughout the calculation terms small in the sense $M^{2} \ll s, t, u \ll m^{2}$ have been neglected. Some details on the actual calculation are given in appendix B.

The result for the one-loop, longitudinally polarized four-W amplitude is

$$
\begin{aligned}
& A_{\mathrm{wwww}}=\frac{i \pi^{2} g^{4}}{M^{4}}\left[\delta_{a b} \delta_{c d}(\right.-\frac{1}{36} s t-\frac{1}{36} t^{2}+\frac{5}{144} s^{2}+\frac{9}{32} s^{2} B_{0}+\frac{1}{32} g_{\mathrm{u}}^{2} s^{2} B_{0} \\
&-\frac{1}{32} s^{2}\left(1+g_{\mathrm{u}}^{\prime 2}\right) \log \frac{s}{m^{2}}-\left(\frac{1}{96} s t+\frac{1}{48} t^{2}\right) \log \frac{t}{m^{2}} \\
&\left.-\left(\frac{1}{96} s u+\frac{1}{48} u^{2}\right) \log \frac{u}{m^{2}}\right) \\
&\left.+\delta_{a c} \delta_{b d}\{s \rightarrow u, t \rightarrow s, u \rightarrow t\}+\delta_{a d} \delta_{b c}\{s \rightarrow t, t \rightarrow u, u \rightarrow s\}\right],
\end{aligned}
$$

$B_{0}=\pi / \sqrt{3}-2$. The $\log$ is always of the absolute value. The absorptive part of the amplitude is not shown here. The term with $g_{\mathrm{u}}$ is the contribution of the U-particle 

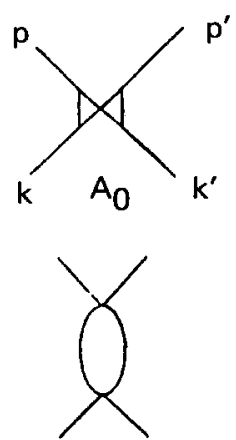

$\mathrm{A}_{3}$

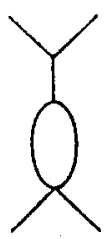

$\mathrm{R}_{3}$

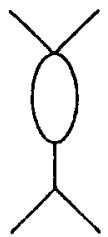

$\mathbf{R}_{4}$

$\mathrm{R}_{5}$

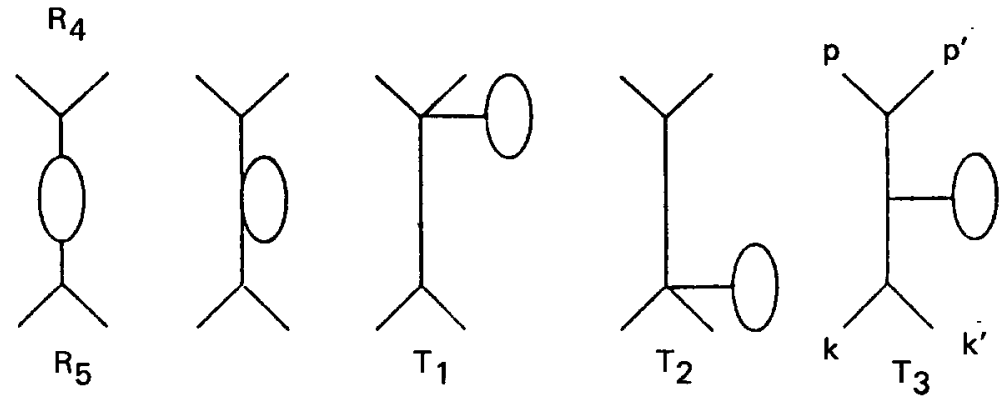

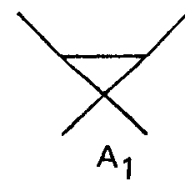

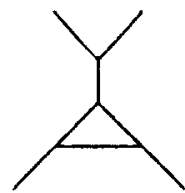

$\mathrm{R}_{1}$
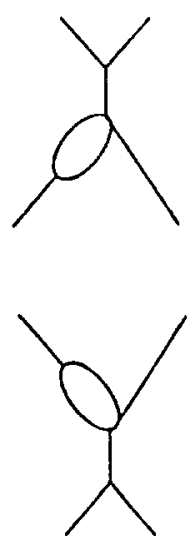

$\mathrm{T}_{1}$
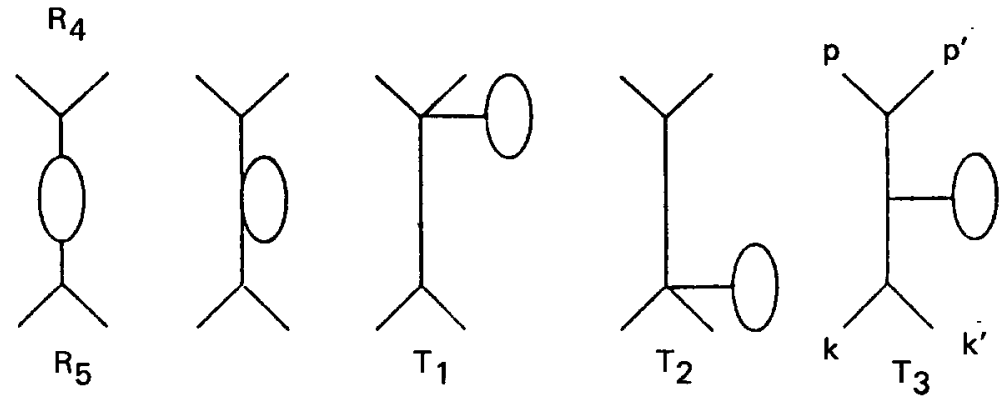
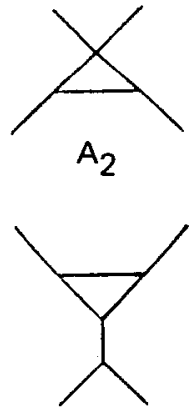

$\mathrm{R}_{2}$
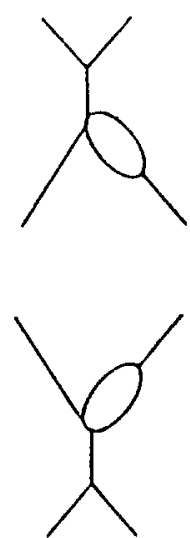

Fig. 3. Topologies of contributing diagrams.

if $m_{\mathrm{u}}=m$, while the term with $g_{\mathrm{u}}^{\prime}$ shows the contribution if $m_{\mathrm{u}}=M$. The dispersive calculation mentioned in the beginning of this section gives the result

$$
\frac{i \pi^{2} g^{4}}{M^{4}} \delta_{a b} \delta_{c d}\left(-\frac{1}{32} s^{2} \log \frac{s}{m^{2}}\right)+\text { terms without } \log (s) .
$$

The result for the four- $\varphi$ amplitude, together with the subtraction terms for that 


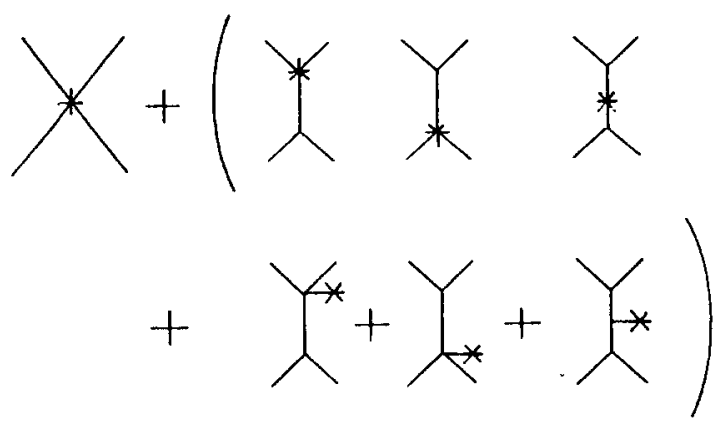

Fig. 4. Structure of counterterms.

four- $\varphi$ case gives exactly the same result as above. The subtraction terms are actually quite different, and there is, for example, no clear relation between the results for certain diagrams (like the topologies $R_{1}-R_{5}$ ) for the four-W and four- $\varphi$ cases.

\section{Discussion of the result}

The results of the previous section show dependence of the four-W amplitude on the U-particle. Thus the resulting terms depend on the details of the limit of large Higgs mass. As such, at this moment, they must be considered ambiguous. There simply is more than one theory. A similar fact has been noted before, in the investigation of two-loop corrections to the $\rho$-parameter. There the ambiguity seems to appear for terms proportional to the Higgs mass squared, but no explicit demonstration as done here with the U-particles has been given.

If, conservatively, we assume no physically observable U-particles (i.e., $m_{\mathrm{u}}=m$ ) then the logarithmic terms appear to be well determined in the sense that they are U-independent. For large Higgs mass they are the leading terms.

Consider now the case that the isospin indices $a=b$ and $c=d$, but $a \neq c$. Also, ignore terms other than logarithms. The ratio of the one-loop amplitude and the tree amplitude is then

$$
R=\frac{A_{1}}{A_{0}}=-\frac{\alpha_{\mathrm{W}}}{\pi M^{2} s}\left(\frac{1}{32} s^{2} \log \frac{s}{m^{2}}+\left(\frac{1}{96} s t+\frac{1}{48} t^{2}\right) \log \frac{t}{m^{2}}+\left(\frac{1}{96} s u+\frac{1}{48} u^{2}\right) \log \frac{u}{m^{2}}\right)
$$

with $\left(\theta_{\mathrm{w}}\right.$ is the weak mixing angle)

$$
\alpha_{\mathrm{W}}=\frac{g^{2}}{4 \pi} \simeq \frac{1}{30}=\frac{1}{137} \frac{1}{\sin ^{2} \theta_{\mathrm{w}}} .
$$


In case that $t=-s$ (and $u=0$ ) one has

$$
R=-\frac{\alpha_{\mathrm{W}} s}{\pi M^{2}} \frac{1}{24} \log \frac{s}{m^{2}} .
$$

Remember that this equation has been derived in the approximation $M^{2} \ll s \ll m^{2}$. With $M \simeq 80 \mathrm{GeV}, s \sim(400 \mathrm{GeV})^{2}$ and $m \sim 2 \mathrm{TeV}$ that might appear reasonable. In that case we find

$$
R=+0.04 \text {. }
$$

This is still quite small. It should be noted that the unitarity limit of Lee et al. obtains in the limit of large $s$, with $s \gg m^{2}$. Our equations do not apply in that limit.

On the bases of the above it appears that for energies below $\frac{1}{2} \mathrm{TeV}$ the corrections to the WW scattering amplitude should remain in the $10 \%$ region for Higgs masses up to quite high values. Of course, we do not know what higher-order radiative corrections will do; in fact, we run already into some ambiguities at the one-loop level.

\section{Appendix A}

In the table below the Feynman rules are given.

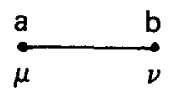

$$
\frac{\delta_{a b} \delta_{\mu \nu}}{k^{2}+M^{2}-i \epsilon} \text { W-propagator }
$$

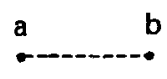

$$
\frac{\delta_{a b}}{k^{2}+M^{2}-i \epsilon} \text { Higgs ghost propagator }
$$

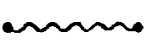

$$
\frac{1}{k^{2}+m^{2}-i \epsilon} \text { physical Higgs propagator }
$$

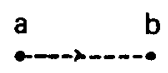

$$
\frac{\delta_{a b}}{k^{2}+M^{2}-i \epsilon} \text { F-P ghost propagator }
$$

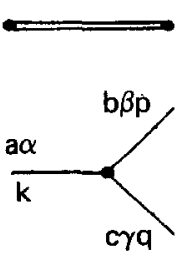

$$
\frac{1}{k^{2}+m_{u}^{2}-i \epsilon} \mathrm{U} \text {-particle propagator }
$$

$$
-i g \epsilon_{a b c}\left[\delta_{\alpha \gamma}(k-q)_{\beta}+\delta_{\beta \gamma}(q-p)_{\alpha}+\delta_{\alpha \beta}(p-k)_{\gamma}\right]
$$

Yang-Mills three-W vertex 


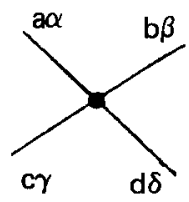

$$
\begin{gathered}
-g^{2}\left[\epsilon_{g d c^{\epsilon b a}}\left(2 \delta_{\alpha \gamma} \delta_{\beta \delta}-\delta_{\alpha \delta} \delta_{\beta \gamma}-\delta_{\alpha \beta} \delta_{\gamma \delta}\right)\right. \\
\left.+\epsilon_{g d b} \epsilon_{g c a}\left(2 \delta_{\alpha \beta} \delta_{\gamma \delta}-\delta_{\alpha \delta} \delta_{\gamma \beta}-\delta_{\alpha \gamma} \delta_{\beta \delta}\right)\right]
\end{gathered}
$$

Yang-Mills four-W vertex

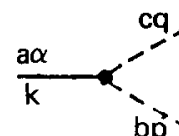

$$
\frac{1}{2} i g \epsilon_{a b c}(p-q) \alpha
$$

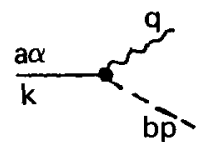

$$
\frac{1}{2} i g \delta_{a b}(p-q)_{\alpha}
$$<smiles>CC(C)(C)C(C)(C)C</smiles>

$$
-\frac{1}{2} g^{2} \delta_{a b} \delta_{c d} \delta_{\alpha \beta}
$$

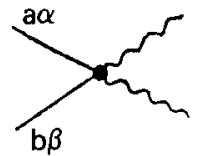

$$
-\frac{1}{2} g^{2} \delta_{a b} \delta_{\alpha \beta}
$$

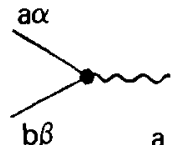

$$
-g M \delta_{a b} \delta_{\alpha \beta}
$$<smiles>[2H]C([18OH])CCC</smiles>

$$
2 r M g \delta_{a b}, \quad r=m^{2} / 4 M^{2}
$$

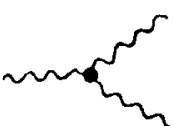

$$
-6 r M g
$$<smiles></smiles>

$$
-r g^{2}\left(\delta_{a b} \delta_{c d}+\delta_{a c} \delta_{b d}+\delta_{a d} \delta_{b c}\right)
$$

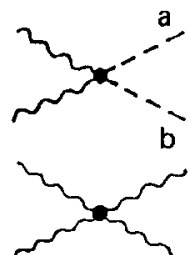

$-r g^{2} \delta a b$

$$
-3 r g^{2}
$$




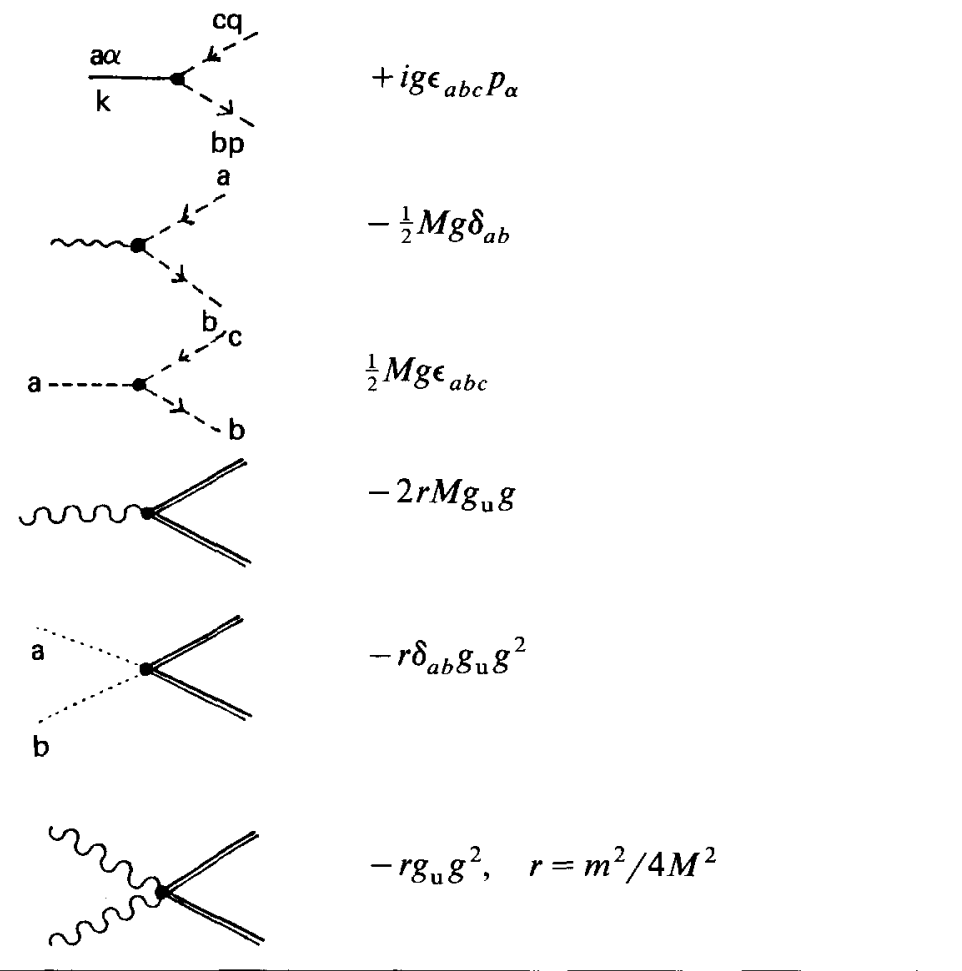

\section{Appendix B}

\section{CALCULATIONAL DETAILS}

The calculation was done with the help of the algebraic manipulation program SCHOONSCHIP. Given the topologies and the Feynman rules it generated all diagrams. Certain elementary tricks were used to evaluate the diagrams in the limit $m^{2} \gg s, t, u \gg M^{2}$. For the WW scattering case the polarization vectors were taken to be proportional to their momentum [e.g., $e_{\alpha}(k)=k_{\alpha} / M$ ].

The basic trick used is to expand Higgs propagators:

$$
\frac{1}{(q+p)^{2}+m^{2}}=\frac{1}{q^{2}+m^{2}}\left[1-\frac{2 q p+p^{2}}{q^{2}+m^{2}}+\left(\frac{2 q p+p^{2}}{q^{2}+m^{2}}\right)^{2} \cdots\right]
$$


where $q$ is the loop momentum to be integrated over. A product of the form

$$
\frac{1}{\left(q^{2}+M^{2}\right)\left(q^{2}+m^{2}\right)^{l}}=\frac{1}{\left(m^{2}-M^{2}\right)^{l}} \frac{1}{q^{2}+M^{2}}+\cdots+\frac{1}{M^{2}-m^{2}} \frac{1}{\left(q^{2}+m^{2}\right)^{l}}
$$

can be rationalized as shown. Also products of the form

$$
\frac{1}{\left(q^{2}+m^{2}\right)\left[(q+p)^{2}+M^{2}\right]}
$$

can be worked out this way: first shift the loop momentum $q^{\prime}=q+p$ and then do the Higgs propagator expansion.

With this technique any mixed occurrence of Higgs and non-Higgs propagators can be eliminated. As it happens, for the $\varphi-\varphi$ scattering amplitude at most two-point functions remain, always discarding nonleading terms for $m^{2} \gg s, t, u \gg$ $M^{2}$. Nonleading terms are actually easily recognizable by the explicit occurrence of factors $M^{2}$. To determine how far to expand the Higgs propagators is more complicated; in the actual calculation terms up to $1 /\left(q^{2}+m^{2}\right)^{6}$ occurred (possibly even higher, but if so they cancelled out).

\section{References}

[1] M. Veltman, Acta Phys. Polon. B8 (1977) 475

[2] D.A. Dicus and V.S. Mathur, Phys. Rev. D7 (1977) 3111

[3] B.W. Lee, C. Quigg and H.B. Thacker, Phys. Rev. Lett. 38 (1977) 883; Phys. Rev. D16 (1977) 1519

[4] M.B. Einhorn, Nucl. Phys. B246 (1984) 75

[5] Part of the results of this paper were found before: R. Akhoury and Y.-P. Yao, Phys. Rev. D25 (1982) 3361

[6] While preparing this article a preprint on the same subject appeared: S. Dawson and S. Willenbrock, Phys. Rev. Lett. 62 (1989) 1232

[7] M. Chanowitz and M.K. Gaillard, Nucl. Phys. B261 (1985) 379; H. Veltman, to be published

[8] J. Van Der Bij and M. Veltman, Nucl. Phys. B231 (1984) 205

[9] R. Bouamrane, private communication 